\title{
Towards for analyzing alternatives of Interaction Design Based on Verbal Decision Analysis of User Experience
}

\author{
doi:10.3991/ijim.v4i2.1239 \\ Marília Mendes ${ }^{1}$, Ana Lisse Carvalhoํㅡㄹ Elizabeth Furtado1, 2, Placido Rogerio Pinheiro1, 2 \\ ${ }^{1}$ University of Fortaleza (UNIFOR, MIA), Fortaleza, Brazil \\ ${ }^{2}$ University of State of Ceará (UECE), Fortaleza, Brazil
}

\begin{abstract}
In domains (as digital TV, smart home, and tangible interfaces) that represent a new paradigm of interactivity, the decision of the most appropriate interaction design solution is a challenge. HCI researchers have promoted in their works the validation of design alternative solutions with users before producing the final solution. User experience with technology is a subject that has also gained ground in these works in order to analyze the appropriate solution(s). Following this concept, a study was accomplished under the objective of finding a better interaction solution for an application of mobile TV. Three executable applications of mobile TV prototypes were built. A Verbal Decision Analysis model was applied on the investigations for the favorite characteristics in each prototype based on the user's experience and their intentions of use. This model led a performance of a qualitative analysis which objectified the design of a new prototype.
\end{abstract}

Index Terms-Human Computer Interaction, Operational Research, Mobile Digital Television, Verbal Decision Analysis, Interaction Design.

\section{INTRODUCTION}

There are some facts which motivated us to have an assumption that applications for Digital TeleVision (DTV) and for mobile devices will be very useful in the next years to users access a huge of services (internet, Learning, Health and Government). First, some nations are preparing their broadcast structure. In UK, now 70\% houses have DTV, 100\% in 2012 [4]. Many network operators (such as in Europe, USA, Japan, Korea and Canada) have started to broadcast TV on handhelds. Second, in emergent countries, a great part of the population has a TV in their homes and uses cell phones [5], but only fews have computers and access to the Internet. Therefore designing usable interactive applications for TV (called iTV applications) is a need to motivate people to use them. Since the DTV represents a new paradigm to interaction design, designers don't have enough knowledge concerning a new domain or technology [19]. There are several practices that designers can apply in order to reduce this difficult. There are many HCI works that give suggestions about the way as professionals should apply user-centered practices when designing and analyzing iTV applications ([12][11]).

Recent researches in HCI are moving from a perspective based on predicting users' behavior (as cognitive features) to a perspective based on observing and understand- ing the users' behavior (as their experiences [10] and emotions [6]). During workshops [20], we could realize that in organizations, as Microsoft, anthropologists and ethnographers are working with usability experts and project managers in order to obtain data related to the following questions: what do the people want to experience? What should the experience feel like for them? Positive and negative comments are obtained from users when describing their experience using software, for instance. Taking into account the users' preferences is an action that has also gained ground in these works when designers are analyzing the better fitting solution(s). Participants of a project must accomplish changes in functionalities from users' contributions for improving the final version of the system. However, the traditional processes of evaluation are quite strict and not flexible to the emergence of new project alternatives and new ways of considering these alternatives. For example, it is typical to find the following scenario: designers evaluate two or three interface solutions applying usability tests, and choose one to implement. At traditional means, usability tests are applied to all alternatives. This work goes beyond the evaluation of this traditional view, by allowing designers to focus on only some criteria of the presented alternatives and by giving them the possibility to think about a new option.

In our approach, the evaluation process is conducted in three steps: first, the designers project high fidelity prototypes with characteristics that want to assess; second, designers carry out the usability tests and analyze the interaction users-TV content shown through each alternative of user's interfaces under the light of user's experience criteria (as users' preferences, their familiarity with technology) and; third, they organize their subjective questions by applying verbal decision analysis in order to define the best characteristics selected by the users during the test process. As a result of this procedure a new alternative of Prototype for Mobile Television Applications could be produced.

We have chosen to apply the Verbal Decision Analysis strategy on the purpose of organizing the usability tests results with sophisticated interactive applications. The reason was that applying to problems which have qualitative nature and difficulty to be formalized, called unstructured [7], may help designers to understand and organize their subjective questions.

The main challenge of this paper is to demonstrate the odds of the construction of a new prototype (a design alternative) starting with the users' opinions, collected 
through usability tests and classified through a computational method like multicriteria model.

We do this, by integrating two different areas (HCI and OR - Operational Research) when we describe an approach for evaluating the Interaction design in a subjective perspective of OR. This approach is co-evolutionary because the evaluation process can restart (as many times as the designers want) being all the design alternatives used again by the same or similar sets of users by feeding the design of a final version.

In this paper, we first present the definition of the prototypes of a Mobile Digital Television Application we developed and implemented. Then we discuss about Verbal Decision Analysis. In the fourth Section, we show a new interaction project. Lastly, some conclusions and indications for future works are provided.

\section{DEFinition OF THE PROTOTYPES OF A Mobile DigitAL TELEVISION APPLICATION}

We had an assumption that a mobile TV application should have the same interaction purpose that an application for DTV. When designing and evaluating a mobile TV application for a specific project being executed, our first impulse was to develop for mobile TV with the same functionalities already developed for DTV, being sure to make the appropriate mapping of visual information between the devices. However we knew new services could be developed to support the users when interacting on movement. Despite that our scenario was the following: the end-users who were involved in this project did not have any experience lived with mobile TV, and we did not have any mobile TV application available to show them as example. Then we decided to apply the following strategy to do the elicitation and validation of new users' requirements. First at all, we looked for existing works in mobile TV to know their main functionalities. Then we analyzed each functionality previously implemented for DTV to verify if it should still be considered and to define how to consider it. Then we implemented one simple visual prototype in order to provide the users a generic idea about its possible usefulness (such as to see movie, to interact with it, to communicate with others, etc).

During a meeting session with the four stakeholders (such as two usability experts, one designer, and one programmer) eight volunteer end-users were presented the prototype in a PDA Palm OS. Each user received one Palm and manipulated the prototype for a period of 20 minutes, performing some non predefined scenarios. They talked one with other when they were not able to do any task. After this period, we asked them about the problems they had experimented as well as the needs they had with respect to their current cell phones and their expectations of mobile services. Some of the most relevant users' comments obtained in terms of interaction design were: 1) when we open a TV we see immediately a channel, when interacting with this mobile application, we need to look for it in the menu options, 2) it was unlikely that mobile TV would be watched in a place where it was not safe, 3) we could only the see the TV services of our interest.

At a certain moment we noticed users familiarized with the iTV applications inspected to interact of the same way, with the same look and feel, even though the RC could not be used. This fact made us to investigate our assumption that the users' experience with technology influences in their preferences for an interaction solution. For this, we defined and implemented different candidate solutions of navigation across the screens, which will be shown as follow.

We used different navigation patterns to design the executable prototypes of this mobile application.

Some features that differentiate applications for DTV and for mobile devices were considered when designing the solutions. These features are: DTVs are used to access TV services at home requiring a (Set-Top-Box) STB to store the applications that implement these services. The existing STBs have capability very limited. Mobile devices are used to access TV services anywhere, and the iTV applications are download directly in the device. In mobile devices, input typically is made using a stylus or finger on a touch sensitive display. In DTV, it is made using the Remote Control (RC). Although applications for DTV have less limitation to place TV information on their screen than applications for mobile devices do, the RC does not allow the direct manipulation on the interaction elements.

As this experiment focused on the users' experience related to the navigation and selection tasks, these differences (mainly the size of screen of the devices and their input styles) were considered in three mobile prototypes. Each prototype presented a different navigation pattern that usually includes icons, links and control of pages to allow the user to perform the navigation and selection tasks. The users access TV services by categories represented by icons or links. For each category, users can navigate through the options to choose a service by selecting the desired service in order to view it (use it then close it).

The three mobile prototypes were the following:

- In the first solution, the designers kept the same style guide of the iTV application (see Figure 1). The most important decisions concerning this pattern were [14]: (1) the navigation arrows, which continued on the bottom and the top with the numeration of the pages in order to inform users that there are more categories of services available (see Figure 1 left side); (2) a navigation arrow, which was included to return to the previous page (see Figure 1 right side); and (3) the navigation bar, which was preserved but without the colored options, because the interaction is made of through the stylus.

- In the second solution, the designers looked for the consistence with other PDA applications and the main change was the following: the navigation arrows to navigate among the categories were substituted by a scroll bar, which is typical in the other PDA applications, and no control to divide the pages was done (see Figure 2, left and right side).

- In the third solution, the style guide was a little bit similar to desktop applications. The designer decided to represent all the TV services as menu options displayed in toolbar located on the top of the screen. In the middle of the screen, the image of option selected can be showed (see Figure 3, left and right side). 

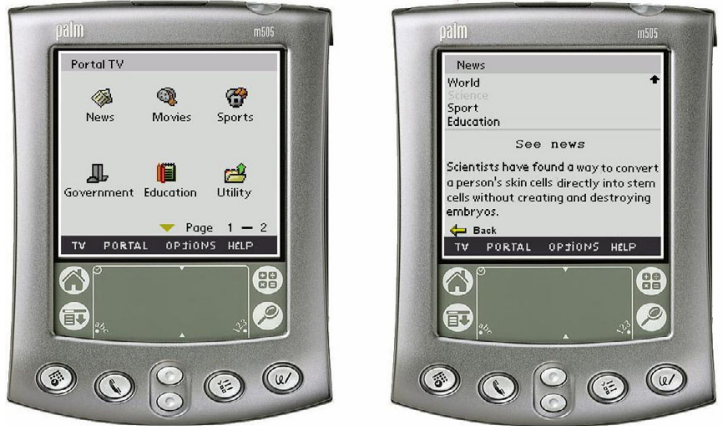

Figure 1 - Prototype 1 . Similar to TVD applications
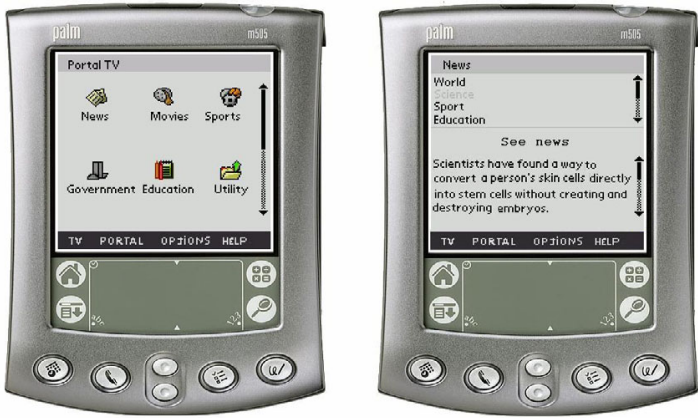

Figure 2 - Prototype 2. Similar to Palm applications
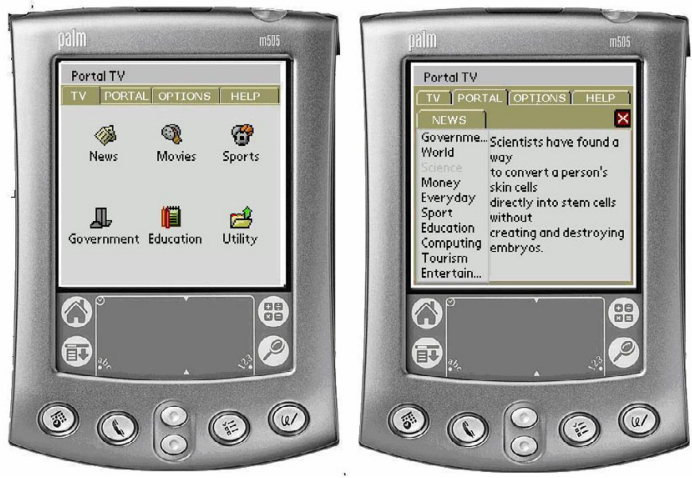

Figure 3 - Prototype 3. Similar to Desktop applications

The scenarios and the questions applied were defined from some hypotheses, that were previously elaborated taking into account the interaction user-applications and the context of use (as the environment, the TV content shown through the user interfaces, the users experience, their emotions, etc.).

The hypotheses were the following:

- Hypothesis 1: The evidence of the of the interface functions facilitates the use and influences in the effort spent by the user to localize himself/herself in the application. We consider here the design decisions that resulted in functions are easily found by the users;

- Hypothesis 2: The user experience with applications which have similar ways of navigation will influence the choice of an interface. Aspects such as facility of use and, accuracy of an interface and user familiarity are included in this hypothesis;

- Hypothesis 3: The locomotion of the user while manipulating the device will influence in the choice of the interface. This hypothesis refers to design decisions that result in less precision to navigate between the op- tions and screens facilitate the navigation of a person while manipulating the application;

- Hypothesis 4: The involvement with the content influences in the user's choice, so that, if the content is interesting, it may be decisive for the user to choose the interface. This hypothesis refers to the holistic evaluation view: when the user uses an interface that has a content which attracts him, s/he will prefer this interface;

- Hypothesis 5: The emotion felt by the user when using the interface exercises a considerable influence in the choice. Aspect such as the user feeling states as pleasure in interactive experiences is considered in this hypothesis.

The hypotheses helped to identify what criteria could bring implication for choosing one specific solution or for choosing just some attributes belonging to the existing solutions. The investigation of this implication was made from the results of the tests [8] that are entered as data for the multi-criteria model used in this paper. Next we present a summary of the ZAPROS III method in order to get a better understanding on its implementation. After we will present how this investigation takes part.

\section{VERBAL DECISION ANALYSIS}

The ZAPROS III method belongs to the Verbal Decision Analysis - VDA framework. It combines a group of methods that are essentially based on a verbal description of decision making problems. It was developed with the aim of ranking given multicriteria alternatives, which makes it different from other verbal decision making, such as ORCLASS [15], and PACOM mainly due to its applicability. The Verbal Decision Analysis supports the decision making process by verbal representation of the problem [16]. It can be applied to problems with the following characteristics [17]: the decision rule must be developed before the definition of alternatives; there are a large number of alternatives; criteria evaluations can only be established by human; the graduations of quality inherent to the criteria are verbal definitions that represent the subjective values of the decision maker.

The decision maker is the key element of multicriteria problems and all necessary attention should be given in order to have well formed rules and consistent and correctly evaluated alternatives. Thus, the categorization of preference will be adequately obtained according to the principle of good ordering established by Zorn's lemma [18].

The application of the method ZAPROS III to modeling problem can be applied following this three-step procedure:

- Elicitation of Criteria and their Values: Once the problem is defined, the criteria related to the decision making problem are elicited. Quality Variations (QV) of criteria are established through interviews and conversations with specialists in the area and decision makers;

- Organization of the Ordinal Scales of Preference: An ordinal scale of preference for quality variations for two criteria is established based on pair wise comparisons. The preference between these two criteria is chosen according to the decision maker and the ob- 
tained scales of preferences are denominated Joint Scale of Quality Variation (JSQV) for two criteria;

- Comparisons of Alternatives: The ranking of the alternatives is constructed by comparisons between pairs of alternatives.

By going through these phases, a problem modeled on the ZAPROS III method results in a display of alternatives [9]. The display gives us a quantitative notion of the order of preference, in an absolute form (in relation to all possible alternatives) and also a relative form (in relation to a restricted group of alternatives). The following is the modeled case study.

\section{Evaluation of Alternatives By ApPlying the ZAPROS III METHOD}

The specialists urged to analyze the aspects that had the greatest influence in the choice of a determined interface project. Then they established verbally some criteria for the implemented prototypes of mobile TVD applications as soon as the possible values for each criterion.

In table 1, the values are shown for the criteria directed to the aspects on which the definition of the influence among the standards is based. For instance, for functions evidence criterion there are three possible values related to the difficulty of the user for identifying the system functionalities.

Table 1 - Criteria and associated values

\begin{tabular}{|c|c|c|}
\hline $\begin{array}{l}\text { Rep- } \\
\text { resen- } \\
\text { tation }\end{array}$ & Criteria & Values \\
\hline A & $\begin{array}{l}\text { Functions } \\
\text { Evidence }\end{array}$ & $\begin{array}{l}\text { A1. There was no difficulty on iden- } \\
\text { tifying the system functionalities; } \\
\text { A2. There were some difficulties on } \\
\text { the system functionalities' identifica- } \\
\text { tion; } \\
\text { A3. It was hard to identify the sys- } \\
\text { tem functionalities }\end{array}$ \\
\hline B & $\begin{array}{l}\text { User's } \\
\text { familiarity } \\
\text { with a de- } \\
\text { termined } \\
\text { technology }\end{array}$ & $\begin{array}{l}\text { B1. No acquaintance was required } \\
\text { with similar applications of a deter- } \\
\text { mined technology; } \\
\text { B2. It was required little user's ac- } \\
\text { quaintance with applications of a } \\
\text { given technology; } \\
\text { B3. The manipulation of the proto- } \\
\text { type was fairly easy when the user } \\
\text { was proverbial with similar applica- } \\
\text { tions. }\end{array}$ \\
\hline $\mathrm{C}$ & $\begin{array}{l}\text { User's } \\
\text { locomotion } \\
\text { while ma- } \\
\text { nipulating } \\
\text { the device }\end{array}$ & $\begin{array}{l}\text { C1. The user was not hindered in } \\
\text { any way when manipulating the } \\
\text { prototype while moving; } \\
\text { C2. The user was occasionally con- } \\
\text { fused when manipulating the proto- } \\
\text { type while moving; } \\
\text { C3. The spatial orientation of the } \\
\text { application was hindered when the } \\
\text { user was moving. }\end{array}$ \\
\hline D & $\begin{array}{l}\text { Content } \\
\text { Influence }\end{array}$ & $\begin{array}{l}\text { D1.There was no influence of con- } \\
\text { tent on choosing the interface; } \\
\text { D2.The content exerted some influ- } \\
\text { ence on choosing the interface; } \\
\text { D3.The content was decisive on } \\
\text { choosing the interface. }\end{array}$ \\
\hline $\mathrm{E}$ & User's & E1. The user felt fine (safe, modern, \\
\hline
\end{tabular}

\begin{tabular}{|c|c|}
\hline $\begin{array}{c}\text { feeling } \\
\text { states }\end{array}$ & $\begin{array}{l}\text { comfortable, etc.) when using the } \\
\text { interface; }\end{array}$ \\
\hline & $\begin{array}{l}\text { E2. The user felt indifferent when } \\
\text { using the interface; }\end{array}$ \\
\hline & $\begin{array}{l}\text { E3. The user felt bad (uncomfort- } \\
\text { able, unsafe, frustrated) when using } \\
\text { the interface; }\end{array}$ \\
\hline
\end{tabular}

The order of preference among the criteria values was established from the results of the tests (observation, questionnaires). For example, it was observed that when the users were moving and trying to execute a task in a determined prototype, they complained that it was difficult to move and manipulate the device at the same time. After the tests, the responses to the questionnaires were gathered and evaluated. Questions like "What prototype did you prefer? And Why?" indicated the order of preference among the project alternatives and also which criteria values were decisive for the choice. The JSQV was gradually elaborated and validated with information from the tests, and resulted in the following sentence:

$$
\begin{gathered}
\mathrm{A} 1 \pi \mathrm{A} 2 \pi \mathrm{B} 1 \pi \mathrm{B} 2 \pi \mathrm{C} 1 \pi \mathrm{E} 1 \pi \mathrm{D} 1 \pi \mathrm{E} 2 \pi \mathrm{D} 2 \pi \mathrm{B} 3 \pi \mathrm{D} 3 \\
\pi \mathrm{C} 2 \pi \mathrm{C} 3 \pi \mathrm{A} 3 \pi \mathrm{E} 3
\end{gathered}
$$

In a simplified way we read the sentence above from A1 to E3 in this way: A1 (No difficulty was found on identifying the system functionalities) is the preferable criteria value, and E3 (The user felt bad (uncomfortable, unsafe, frustrated) when using the interface) is the least desirable because it was not perceived.

The next step of the method was to carry out the comparison of the alternative standards. Each alternative was studied in order to define which criteria value the materialized prototypes. The usability tests also supplied important information on how the users described the alternative standards (for example, the majority of users said that access to content using prototype 3 (three) was quite easy. Three alternatives the most preferred of all were established from this information (presented by preference order):

- Prototype 1 - A2 B1 C2 D1 E2 (Alternative 1);

- Prototype 2 - A2 B3 C1 D1 E1 (Alternative 2);

- $\quad$ Prototype 3 - A2 B1 C1 D1 E2 (Alternative 3).

In a simplify way, the majority of users said for the second prototype that the interaction with the application was not damaged while moving (C1) and that they had fun using it (E1). The applied model was useful for the choice of the most favorite prototypes (details of this multicriteria model application can be obtained in previous works of the authors of this paper [8], [1], [2] and [13]). However it is not enough to choose a single option among the presented three, instead we want to identify the best characteristics (represented by the criteria values) existing in the analyzed prototypes. Next we aim at showing how it is possible to elaborate a new prototype based on the identified characteristics.

\section{ELABORATION OF A NEW INTERACTION PROJECT}

This section we will have three sub-sections. In the first one, we show how the design was done collaboratively 
with the designers, in the second one, we will analyze the values of criteria of each prototype that will be useful to develop a new proposal (project). In the third one, we will design this new project. For the creation of the new project it was considered JSQV and the selection of the best characteristic of each prototype with base on the values of criteria presented by them. To sketch the new interface, a tool was used called SketchiXML. SketchiXML is a multi-platform multi-agent interactive application that enables designers and end users to sketch user's interfaces with different levels of details and support for different contexts of use [3].

\section{A. Collaborative Design}

We conducted a collaborative design session aiming to understand the views of designers on the outcome of the case study, to design a new solution for interaction. For this, we considered the scale JSQV and the selection of preferred features of each prototype based on the values of criteria presented. In this sub-section we explain the methodology applied during the collaborative design.

The collaborative design session lasted an hour and involved a group composed of the designer and analyst (specialist in ZAPROS) who participated in this case study, as well as three other volunteer designers.

The main designer led the session in a lab environment, using a data show, presenting, through explanatory slides, a summary of the case study conducted with the proposed strategy. The results of the strategy (ordering of alternatives and criteria values) were reported and interpreted by the designer and analyst, aiming to explain to three designers invited the meaning of the ranks in the preferences of users. This analysis will be explained in more detail in the next sub-section $B$.

All the participants discussed the characteristics of each alternative. The three designers (Figure 4) found the results of the strategy very interesting and useful to know the preferences of users, extract the best features and improve the weaknesses identified. Through these discussions, a new prototype has been developed collaboratively (Figure 5). The result of the design Collaborative has been consolidated in the design of interaction using the tool SketchiXML which will be explained in sub-section C.

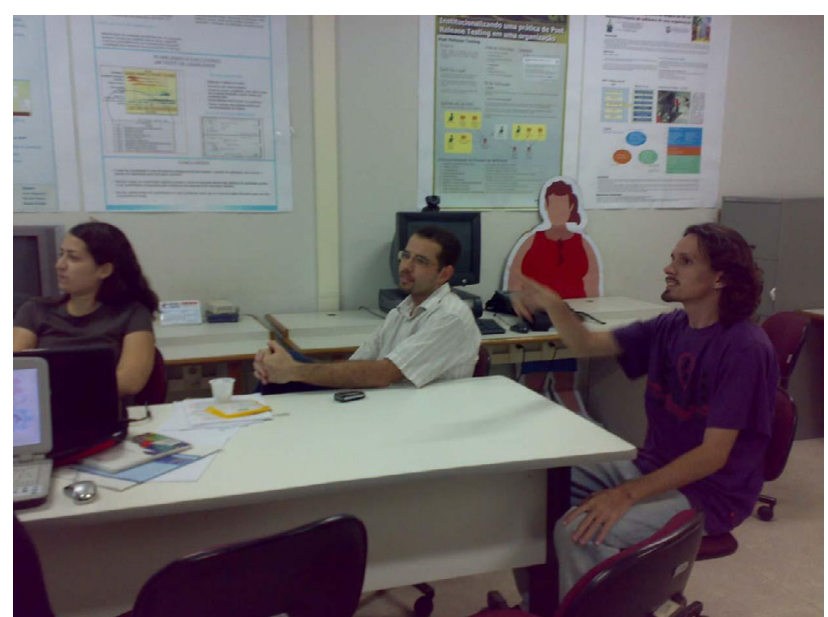

Figure 4 - The three designers to make decisions based on the results of case study

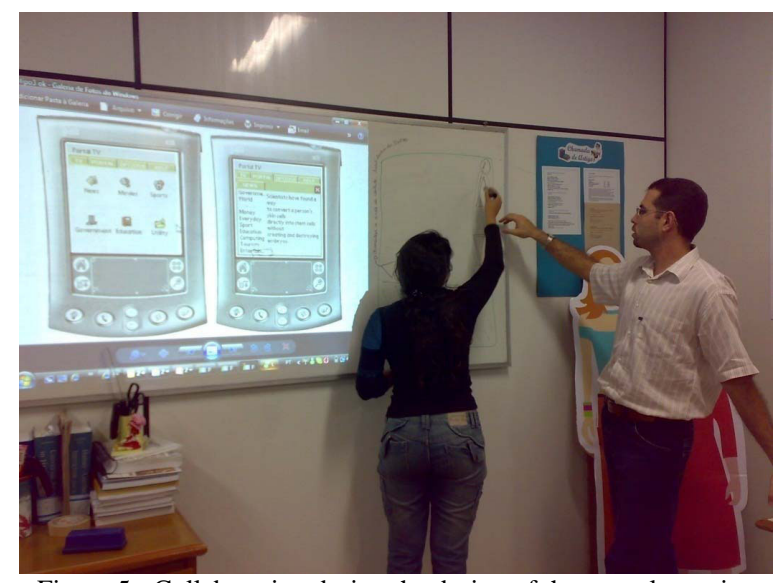

Figure 5 - Collaboration during the design of the new alternative

\section{B. Analysis of the values of criteria of each prototype}

In Figure 6, we can visualize in prominence the values of better rank in the scale JSQV of each prototype. They will be the base for analysis and subsequent elaboration of the new prototype.

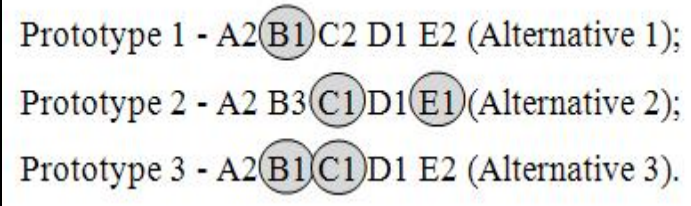

Figure 6 - Selection of the values in agreement with the scale

When a value is repeated in the three prototypes, as the value A2 (that means, the users had some difficulty on identifying the system functionalities), we make use of the same value for the three prototypes.

In the second column, we obtained two different values: B1 and B3. In agreement with the rank, B1 is preferred in relation to $\mathrm{B} 3$, for that reason we will consider the value $\mathrm{B} 1$ that was identified in the prototypes 1 and 3 with the characteristic: no acquaintance is required with similar applications of a determined technology (B1), meaning that it is not necessary to have experiences with a given technology for a good use of the two prototypes.

In the third column, we obtained two different values: $\mathrm{C} 1$ and C2. In agreement with the rank, C1 is preferred in relation to $C 2$, for that reason we will consider the value $\mathrm{C} 1$ that was identified in the prototypes 2 and 3 with the characteristic: the user was not hindered in any way when manipulating the prototype while moving (C1), therefore we can consider in the new prototype, similar navigation ways of the prototypes 2 and 3, like scrolling and tabs. In the fourth column, the value D1 is repeated for the three prototypes. The value says that there was not influence of content on choosing the interface (D1). In the last column, we obtained two different values: E2 and E1. In agreement with the rank, the value E1 is preferred in relation to $\mathrm{E} 2$, for that reason we will consider the value E1 that was identified in the prototype 2 with the characteristic: the user felt fine (safe, modern, comfortable, etc.) when using the interface, therefore we should consider some of the characteristics of the prototype 2 that turned its comfort- 
able use for the tested users.

\section{The new interaction project}

The new prototype (see figure 7) was projected based on analyzes explained in the previous item.

The prototype 1 contains criterion value B1 that is with rank 3 in the final scale, this means that it is very important that the new prototype does not demand any user experience with technology. Hence, we selected the arrow with explanatory label for symbol of the navigation: lateral (returning the previous screen) and of lower or of top (with passage of pages down and upward, respectively).

The third prototype presents besides the value B1, the value $\mathrm{C} 1$, because it makes the manipulation of the prototype easier, when the user is in movement. Therefore, it was necessary also to select some characteristic of this prototype. The selected characteristic was the one that possesses a larger area of contact through the tabs, then a junction of B1 was accomplished with $\mathrm{C} 1$, resulting in an arrow of such adult for navigation. Another selected characteristic of the prototype 3 was the located menu in the superior part of the screen.

The second prototype contains the value E1 as differential, and also contains the characteristic that was taken as advantage in the new prototype: the scrolling bar that was used for navigation interns through the widgets combox for list of title of the news and listbox for the content of the selected news.

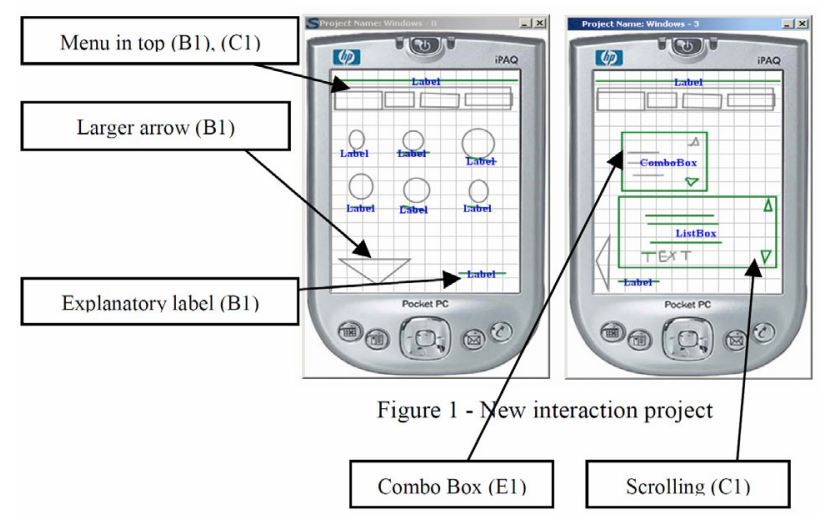

Figure 7 - New interaction project

We expect this proposal to be more adequate according to the following reasons: the use of bigger arrows makes possible the change of screens especially when the users are moving; the use of explanatory labels help the users about the context of the application; the menu located at the superior part of the screen was favored by the user because it makes easy the visualization of the options.

\section{CONCLUSIONS AND FUTURE WORKS}

From this conclusion we described some points that can be classified in HCI and OR areas: In HCI, the points refer to both what to design and evaluate and in OR, they refer to analysis of these design and evaluate through the ZAPROS III multicriteria method.

In HCI area, the points are the followings:

- Designers can produce applications more innovative and adapted to the users' cultural issues if they understand better the technological scenarios lived by the users. Theirs should also provide users with TV content design artifacts rather than just focus on the user interfaces of the iTV applications. Designers should express their ideas, by showing users several entertainment situations they could have by anticipating and proactively perceiving their emotions (feeling states, involvement with the content).

- Users should understand better what they will have at the end of the project. It is common to hear the following phrase: the users don't know what they want. Making the users to explore a possible solution by themselves simulating situations that occur in the real world can be important to designers to feel the users' acceptance.

- Designers should go beyond the usability when doing usability tests with users. Traditionally, the usability of a developed system has been evaluated to assure both its effectiveness (such as the number of successful task completions) and efficiency (such as the time required to complete an interactive task). Recently, these assumptions have been revisited and broadened to embed the concepts of the affective quality theories. The affective aspect (such as users' feeling states, pleasure and their involvement with the content) is particularly relevant in the context of iTV, since entertainment depends strongly on how synergetic the involvement of the viewer with the TV content.

In OR area, the point is:

- The multicrieria method was useful in the elaboration of another design alternative, because it showed a structured model focused on users' preferences and by defining a rank with the best distinct characteristics by the own users in usability tests. This new e final prototype contemplated these best criteria. In our approach, it is not imperative to build a new design alternative or a final prototype. From a more general point of view, some useful lessons about the utility and the usability of similar systems (e.g. user interface guidelines) can be learned and further applied in future projects.

The idea of using sketchiXML for visualization of the new prototype was considered useful because it is a fast way to obtain an initial sketch. In addition, this tool generates the interface specifications in a standard format, as the UsiXML. This format is widely interpreted by interface generation tools that are freely available for use. These tools allow the automatic generation of the final user interface. In our approach, executable prototypes need to be easily obtained and quickly tested by the users in order to support its evolutionary characteristic.

As future works and next steps, we intend to validate this new prototype with the users by performing a second test in order to verify if the prototype is really in agreement with the best suggested characteristics. Using the ZAPROS III multicriteria method, we will include the new developed prototype as another alternative in order to be sure that it is really the best of the fourth alternatives. This iterative feature characterizes the co-evolutionary process of the project

\section{REFERENCES}

[1] A. Carvalho, M. Mendes, P. Pinheiro, E. Furtado, Analysis of the Interaction Design for Mobile TV Applications based on MultiCriteria, International Conference on Research and Practical Issues of Enterprise Information Systems (CONFENIS 2007), October 14-17, Beijing, China. 
[2] A. Carvalho; M. Mendes; E. Furtado; P. Pinheiro. Avaliação de Projetos de Interação para Aplicações de TVD Móvel utilizando Multicritério.. In: XXXIX Symposium of the Brazilian Operational Research Society (XXXIX SBPO), 2007, Fortaleza. XXXIX Symposium of the Brazilian Operational Research Society, 2007.

[3] A. Coyette, J. Vanderdonckt. A Sketching Tool for Designing Anyuser, Anyplatform, Anywhere User Interfaces, Proc. of Interact'2005.

[4] A. Jaokar, T. Fish, Mobile Web 2.0. FutureText. 2006.

[5] Agência Nacional de Telecomunicações. http://www.anatel.gov.br/Tools/frame.asp?link=/biblioteca/release s/2005/release_15_09_2005(2).pdf

[6] D. Norman, Emotional Design. Basic Books. 2004.

[7] H. Simon, A.Newell, Heuristic Problem Solving: The Next Advance in Operations Research, Oper. Res., vol. 6, pp. 4-10, (1958). (doi:10.1287/opre.6.1.1)

[8] I. Tamanini; M. Mendes; A. Carvalho; E. Furtado; P. Pinheiro. A model for mobile television applications based on verbal decision analysis. In: Tarek M. Sobh. (Org.). International Joint Conferences on Computer, Information, and Systems Sciences, and Engineering: 2008, to appear.

[9] J. Figueira, S. Greco, M. Ehrgott,(Eds.), Multiple Criteria Decision Analysis: State of the Art Surveys Series: International Series in Operations Research \& Management Science, Vol. 78, XXXVI, 1045 p, (2005).

[10] J. McCarthy, P. Wright, Technology as experience. MIT.2004.

[11] K. Chorianopoulos, D. Spinellis, User Interface Evaluation of interactive TV: a media studies perspective. Univ. Access Inf Soc. 5: 209-218. 2006. (doi:10.1007/s10209-006-0032-1)

[12] L. Eronen, User Centered Design of New and Novel Products: case digital television. Thesis. Publications in Telecommunications software and multimedia. 2004.

[13] M. Mendes, E. Furtado, Mapeamento De Um Portal De Acesso De Televisão Digital Em Dispositivos Móveis. IHC’2006.

[14] O. Larichev, Cognitive validity in design of decision-aiding techniques, Journal of Multi-Criteria Decision Analysis, 1(3): 127138, (1992). (doi:10.1002/mcda.4020010303)

[15] O. Larichev, H. Moshkovich, Verbal Decision Analysis For Unstructured Problems, Boston: Kluwer Academic Publishers, (1997).

[16] O. Larichev, Ranking Multicriteria Alternatives: The Method ZAPROS III, European Journal of Operational Research, Vol. 131, (2001). (doi:10.1016/S0377-2217(00)00096-5)

[17] P. R. Halmos, Naive Set Theory, Springer, 116 p., (1974).

[18] S. Dow, T. Saponas, Y. Li, J. Landay, External Representations in Ubiquitous Computing Design and the Implications of Design Tools. Proc. DIS'2006. Pp 241-250. 2006.

[19] T. Winograd, T., CS147: Introduction to Human-Computer Interaction, 2006. Stanford, CA. http://cs147.stanford.edu.

\section{AUTHORS}

Marília Soares Mendes has a degree in Computer Science from University of Fortaleza, Ce, Brazil (2006). Master in Applied Informatics from University of Fortaleza, Ce, Brazil (2009). Currently, she is a doctoral student at the Federal University of Ceará, Brazil. She has experience in computer science, with emphasis on research in human computer interaction (HCI), usability and software engineering, working mainly in digital television and mobile devices. (e-mail: mariliamendes@gmail.com).

Ana Lisse Carvalho has a degree in Computer Science from University of Ceará, Brazil (2004), Master in Applied Informatics from University of Fortaleza, Ce, Brazil (2008). Currently, she is a Civil Servant of Federal Office for Data Processing. She has experience in the area of computer science, especially operational research and software engineering. (e-mail: ana.lisse@edu.unifor.br).

Elizabeth Furtado is a Researcher in human computer interaction (HCI) and a Visiting Scholar (a sabbatical study) at HCI laboratory in Stanford University, in 2006. She is a consultant of software quality process. She did her $\mathrm{PhD}$ in Computer Science in 1997 in France. She is Adviser of the graduate and undergraduate students in the Master program of the MIA, University of Fortaleza, Ce, Brazil since 1989, in usability of collaborative learning systems; definition of tools for working with guidelines and patterns in order to generate user interfaces for interactive television (iTV) and definition of scenarios to evaluate iTV applications. (e-mail: elizabet@unifor.br).

Plácido Rogério Pinheiro has a degree in Mathematics from Federal University of Ceará, Brazil in (1979). He is an Electrician Engineer from the University of Fortaleza, Ce, Brazil (1983), with Master degree in Mathematics at the Federal University of Ceará (1990) and PhD in Systems Engineering and Computation, Federal University of Rio de Janeiro, Ce, Brazil (1995). He is currently Professor at the University of Fortaleza. He has experience in the modelling of industrial processes using mathematical programming and multicriteria. His training allows publishing in the area of mathematics, with emphasis on discrete mathematics and combinatorics, working primarily in mathematical programming and multicriteria. (e-mail: placido@unifor.br).

Submitted February $23^{\text {rd }}$, 2010. Published as resubmitted by the authors March 18th, 2010. 